\title{
Designing Right-Turn Vehicle Box as a Supplemental Treatment to Eliminate Conflicts with Pedestrians and Bicycles
}

\author{
Fengxiang Qiao, Po-Hsien Kuo, Qing Li, Lei Yu, Qing Zhu, Ying Li \\ Innovative Transportation Research Institute, Texas Southern University, Houston, Texas, USA \\ Email: qiao_fg@tsu.edu, ckin44@gmail.com, liq@tsu.edu, yu_lx@tsu.edu, qingzhu0223@gmail.com, \\ alee8291@gmail.com
}

Received 5 January 2015; accepted 26 January 2016; published 29 January 2016

Copyright $@ 2016$ by authors and Scientific Research Publishing Inc.

This work is licensed under the Creative Commons Attribution International License (CC BY).

http://creativecommons.org/licenses/by/4.0/

c) (i) Open Access

\section{Abstract}

Field observations illustrated that, right-turn vehicles stopped at various positions when proceeding within the right-turn lanes, while some of them trespassed on the crosswalks with multiple stops. In this case, pedestrians and bikes (ped/bike) are encountered unsmooth and hazardous crossings when right-turn vehicles encroaching their lanes. Meanwhile, this also causes conflicts between right-turn and through vehicles at the crossing street. To better protect ped/bike at crossings with right-turn vehicles, this paper proposes a concept of "right-turn vehicle box" (RTVB) as a supplemental treatment within right-turn lanes. Sight distance, geometric conditions, and behaviors of vehicles and ped/bike are key factors to consider so as to set up the criteria and to design the suitable treatment. A case study was conducted at an intersection pair in Houston, USA to shape the idea of RTVB, together with driving simulator tests under relevant scenarios. The preliminary crosscheck examination shows that the right-turn vehicle box could possibly provide ped/ bike with smoother and safer crossings. In the interim, the safety and efficiency of right-turn operations were also improved. To further validate the effects, implementation studies should be conducted before the RTVB can make its debut in practice. Future works will focus on the complete warrants and design details of this treatment. Moreover, the concept of "vehicle box" could also be transplanted to other places where turning movement(s) needs assistance or improvements.

\section{Keywords}

Right-Turn Vehicle Box, Bicycle, Pedestrian, Right-Turn Treatments, Channelized Right-Turn Lane

How to cite this paper: Qiao, F.X., Kuo, P.-H., Li, Q., Yu, L., Zhu, Q. and Li, Y. (2016) Designing Right-Turn Vehicle Box as a Supplemental Treatment to Eliminate Conflicts with Pedestrians and Bicycles. Journal of Transportation Technologies, 6, 43-59. http://dx.doi.org/10.4236/itts.2016.61005 


\section{Introduction}

The safety at intersections is a big concern in urban traffic operations. During 2012, The United States lost 33,561 people in crashes on roadways, while 8766 were at intersections [1]. This includes 4743 pedestrians and 726 cyclists killed at intersections [2] [3]. For years, in order to eliminate the conflicts between pedestrians and bicycles (ped/bike), and motor vehicle conflicts and to improve the safety as well as the efficiency of the entire intersection operation, tremendous efforts have been proposed on many elements related to the design and management of intersection, such as the configurations for left-turn (e.g. [4]), signal timing (e.g. [5] [6]), proximate driveway placement (e.g. [7] [8]), advanced devices (e.g. [9]). However, few studies were conducted on right-turn treatments (e.g. [10] [11]) for the sake of ped/bike.

Since late 1970s, a Right-Turn-On-Red (RTOR) policy was adopted by the United States to permit right-turn movements in the condition that the driver had stopped and there was no specific prohibition by any traffic sign [12]. However, the RTOR policy could increase the frequency of right-turning crashes, especially the ones involving ped/bike [12]. There are several methods to restrict the use of RTOR in order to promote safety, e.g. placing traffic signs prohibiting RTOR during specified hours and when pedestrians are present [13].

Channelization and refuge islands (or called traffic islands) are typical treatments to delineate right-turn movements [14]. According to the American Association of State Highway and Transportation Officials' (AASHTO) Policy on Geometric Design of Highways and Streets, or called the Green Book, the refuge islands have three primary functions: (1) division, (2) channelization, and (3) pedestrians' refuge [15], which allow high-priority traffic movements within preset control mode, and to separate slow and stopped vehicles from high-speed vehicles [16]. At signalized intersections, the right-turn movement is normally controlled by traffic lights, and sometimes by a yield sign [17].

The applications of the above treatments should have yielded a safe and efficient right-turn operation; however, there are still potential safety problems to be further improved. The right-turn vehicles normally stop at different positions along the right-turn channel while some of them executed more than one stop before completing a turning. Further, many right-turn vehicles could encroach on crosswalks. These unpredictable actions do cause potential conflicts.

The objective of this paper is to propose a new right-turn treatment so as to eliminate potential conflicts, and promote the safety and efficiency of right-turn vehicles as well as the ped/bike operations.

\section{AASHTO Design Guide and Conflicts Identified from Field Observations}

\subsection{ASSHTO Design Guide on Right-Turn Maneuver}

\subsubsection{Clear Sight Triangle}

The clear sight triangle is defined as a specified area clear of obstructions, which could block a driver's view [15]. Figure 1 illustrates a typical triangle of clear sight to view the traffic from the crossing street on the left.

In Figure 1, the dimension " $a$ " represents the distance from the major road along the minor road, and the dimension " $b$ " indicates the distance to conflict for the approaching vehicle the crossing street on the left. The decision point in Figure 1 is where the drivers start to brake for stop in the case that a vehicle on crossing street from left is approaching.

\subsubsection{Sight Distance for Right-Turn Vehicles}

According to the AASHTO Green Book [15], the proper sight distance with appropriate traffic control can greatly reduce the potential vehicular conflicts at intersections. For drivers of stopped vehicles, the sight distance allows a sufficient view to make the decision of when to enter the intersecting road.

The Required Sight Distance (RSD) depends on the basic maneuver of the entering vehicles. For the vehicles turning right into the roadway, the RSD varies with the design speed. For example for urban arterial streets, the RSD is $160 \mathrm{~m}$ for design speed $60 \mathrm{~km} / \mathrm{hr}, 250 \mathrm{~m}$ for $80 \mathrm{~km} / \mathrm{hr}$, and $370 \mathrm{~m}$ for $100 \mathrm{~km} / \mathrm{hr}$ [15].

\subsubsection{Stopping Positions}

Vehicles normally do not stop exactly at the location of stop lines. Observations show that, the distance between the front of a stopped vehicle and the edge of the major-road traveled is $2 \mathrm{~m}(6.5 \mathrm{ft})$ or less [15]. For the average passenger vehicles in the U.S., the distance between the front of the vehicle and the driver's eyes is about $2.44 \mathrm{~m}$ (8 ft) or less [17]. 


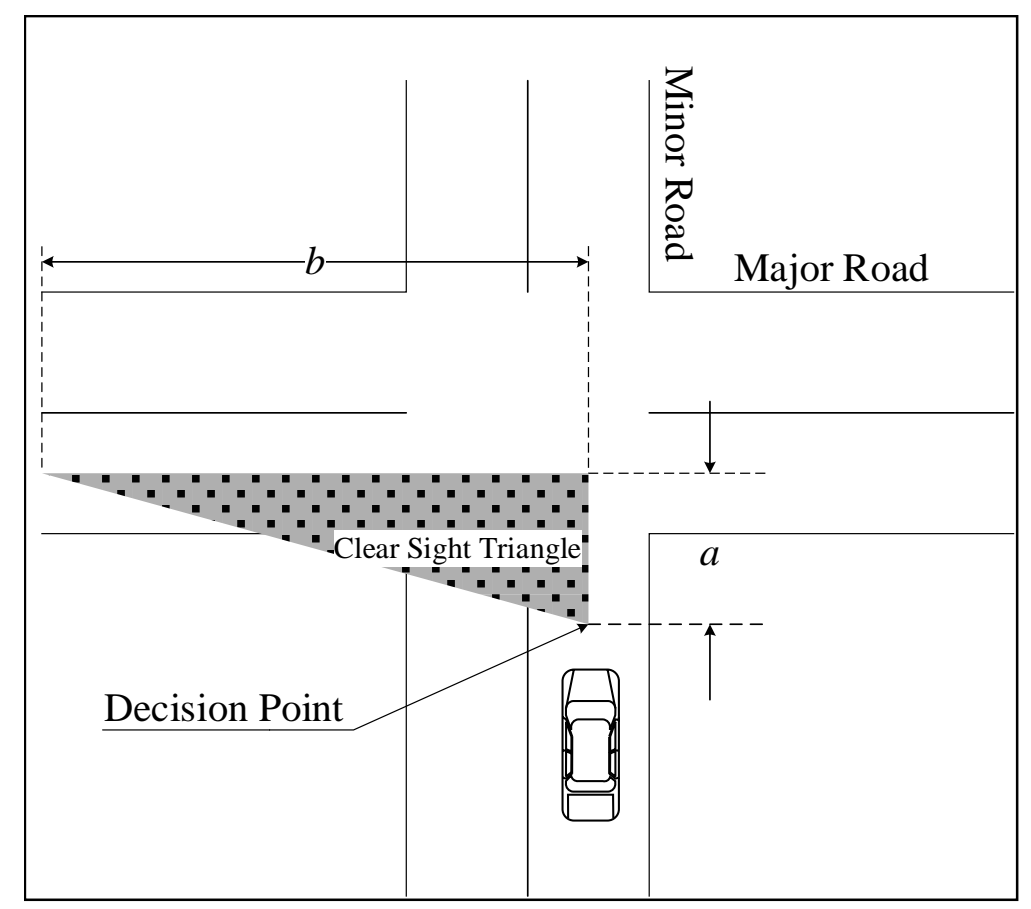

Figure 1. Clear sight triangle for viewing traffic approaching from the left. Source: Exhibit 9-50A from AASHTO’s Green Book [15].

In the AASHTO Green Book [15], the guidelines on right-turn operations are mainly focused on intersections with no right-turn lanes. The guideline on right-turn vehicle movement and its impact to ped/bike within rightturn lanes is not included in the current version.

\subsection{Observations at Four Right-Turn Lanes and the Conflicts with Ped/Bike}

In order to formulate the idea for a special treatment for right-turn vehicles at channels, afield observation was conducted. Couple intersections with exclusive right-lane channels were selected along Bellaire Boulevard, Houston, TX, USA, which intersects the northbound and southbound frontage roads of a toll road called "Beltway 8". The four exclusive right-turn channels were indicated as SB, EB, WB, and NB in Figure 2. Each crosswalk is part of a multi-use path. Motorized traffic volume, local geometric layouts, and non-motorized traffic movements were recorded using three cameras, which were deployed in locations A, B, and C. More specifically, one camera was mounted on the $12^{\text {th }}$ floor of a business building closest to the WB and NB channels (Location C). Other two cameras were placed at grade for the view of SB and EB (Locations A and B). The behaviors of the right-turn vehicles and ped/bike crossings, and potential conflicts were recorded.

The observations were conducted during the morning and afternoon peak hours, from 7 am to 9 am, and $4 \mathrm{pm}$ to $6 \mathrm{pm}$, in four weekdays (Tuesday July 7; Wednesday July 8; Monday July 13; and Tuesday July 14). Totally, 30 hours of video tapes were recorded. Through the careful analysis of the recorded right-turn movement at the intersections, the following features were observed.

\subsubsection{Different Length of Right-Turn Channels}

It was observed that for each right-turn channel, there is a set of refuge islands for pedestrians and bicyclist. The four sets of refuge islands are shaped differently, which result in different length of right-turn channels. It is worth noting that the shortest of the right-turn channel is for NB, which is even shorter than the length of a standard passenger vehicle with $2.1 \mathrm{~m}$. On the other hand, there is no crosswalk observed on the right-turn channel, which means ped/bike may cross the channel from anywhere. Relatively, the design of the right-turn channel for WB and EB are similar to each other, including the shape of refuge islands, the length of right-turn channels, and the location of crosswalks. Further, the space for right-turn vehicles stopping without crossing over the crosswalk is sufficient. 


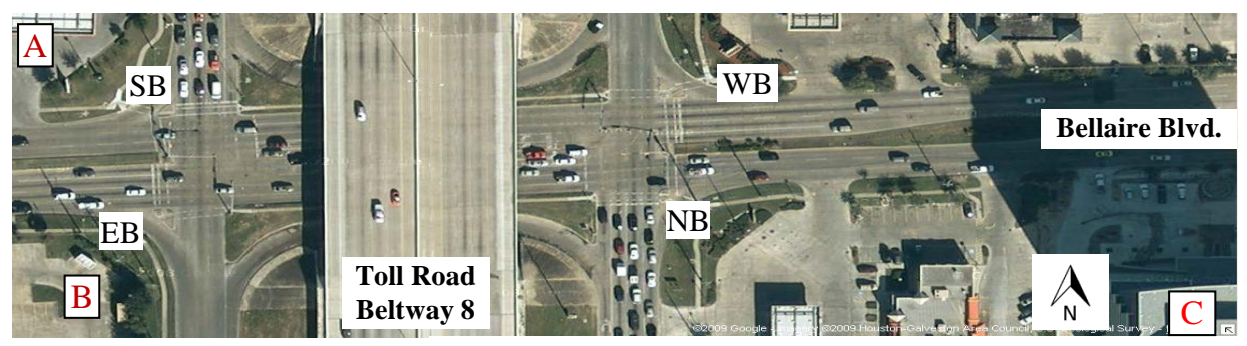

Figure 2. Layout of four observation sites: westbound (WB), southbound (SB), northbound (NB), and eastbound (EB). Three cameras were deployed in three locations indicated with A, B, and C.

\subsubsection{Ten Types of Stopping Positions}

Based on the observation, vehicles didn't stop at a common position within a right-turn channel prior to the action of right-turn. The distribution of stopping positions of right-turn vehicles could be categorized into 10 types, as is shown in Figure 3.

In Figure 3, types 2 and 3 located above a crosswalk are considered as "ideal" situations, as there was no conflict with pedestrians or bicyclists observed. The difference among types 1, 2, and 3 is that, a second vehicle that follows the target vehicle may (type 1) or may not (type 2) stopover the crosswalk (type 1), or there is no following vehicle at that time (type 3). Occupation of crosswalk in type 1 led to a conflict with ped/bike, thereby not being regarded as an "ideal" situation.

Besides, when vehicles' body entirely or partially occupied the crosswalk like type 4 to 7 , conflicts with ped/ bike may take place as well. At the upstream of the crosswalk, vehicles lining up in the stopping positions of types 8 to 10 were regarded as good cases. However, at those positions, drivers might not see the upcoming vehicles from left side of the crossing street.

\subsubsection{Multiple Stopping}

By observations, a certain proportion of vehicles conducted multiple stops before completing a right-turn movement. The statistics of vehicles executed multiple stops are listed in Table 1.

At the right-turn channels on EB and WB, $11 \%$ and $27 \%$ of vehicles performed more than one stop, respectively. This phenomenon was especially obvious on NB, where $44 \%$ of the vehicles executed more than one stop (Figure 4). Multiple stops or yield movements when appropriate are allowed by Chapter 545 of the Texas Transportation Code [18], which however reflects to some extent the complexity of the traffic and/or geometry at the situation.

The distributions of vehicle stops in terms of the ten types of positions were illustrated in Figure 4.

In Figure 4, at most of the right-turn channels (NB, SB, and EB), right-turn vehicles stopped more than one time at different positions. For example at the NB (Figure 4(b)), most right-turn vehicles stopped at types 6 and 7, both intruded the crosswalks.

The WB (Figure 4(a)) is an ideal case, where most of the vehicles stopped at type 3 (fully in front of the crosswalk), no interruption with the designed crosswalk. Only a small portion of right-turn vehicles stopped at type 4, meaning that at those limited cases the vehicles slightly intruded the crosswalk (with their front bumps). The SB is also a good case, where most vehicles stopped at type 8 (behind the crosswalk) without intruding the designed crosswalk.

\subsubsection{Various Type of Conflicts with Ped/Bike}

The right-turn vehicles might encroach on the crosswalk and induced various types of conflicts with ped/bike. The types of conflicts are listed in Table 2.

Due to the right-turn on red police and right-turn channelization, ped/bike crossings could be more difficult within the mixture of high-speed motorized traffic at intersections. Table 2 displays the category of crossing behaviors of ped/bike, including waiting, stop and go, crossing at front of vehicle, crossing at rear of the vehicle, giving up the crossing. The potential conflicts may occur when crossing at front, crossing at rear, and giving up. The potential conflict rate with ped/bike ranges from $16 \%$ to $40 \%$ among the four right-turn channels.

While it is believed that the higher demand of crossing may lead to higher potential conflicts, this phenomenon 


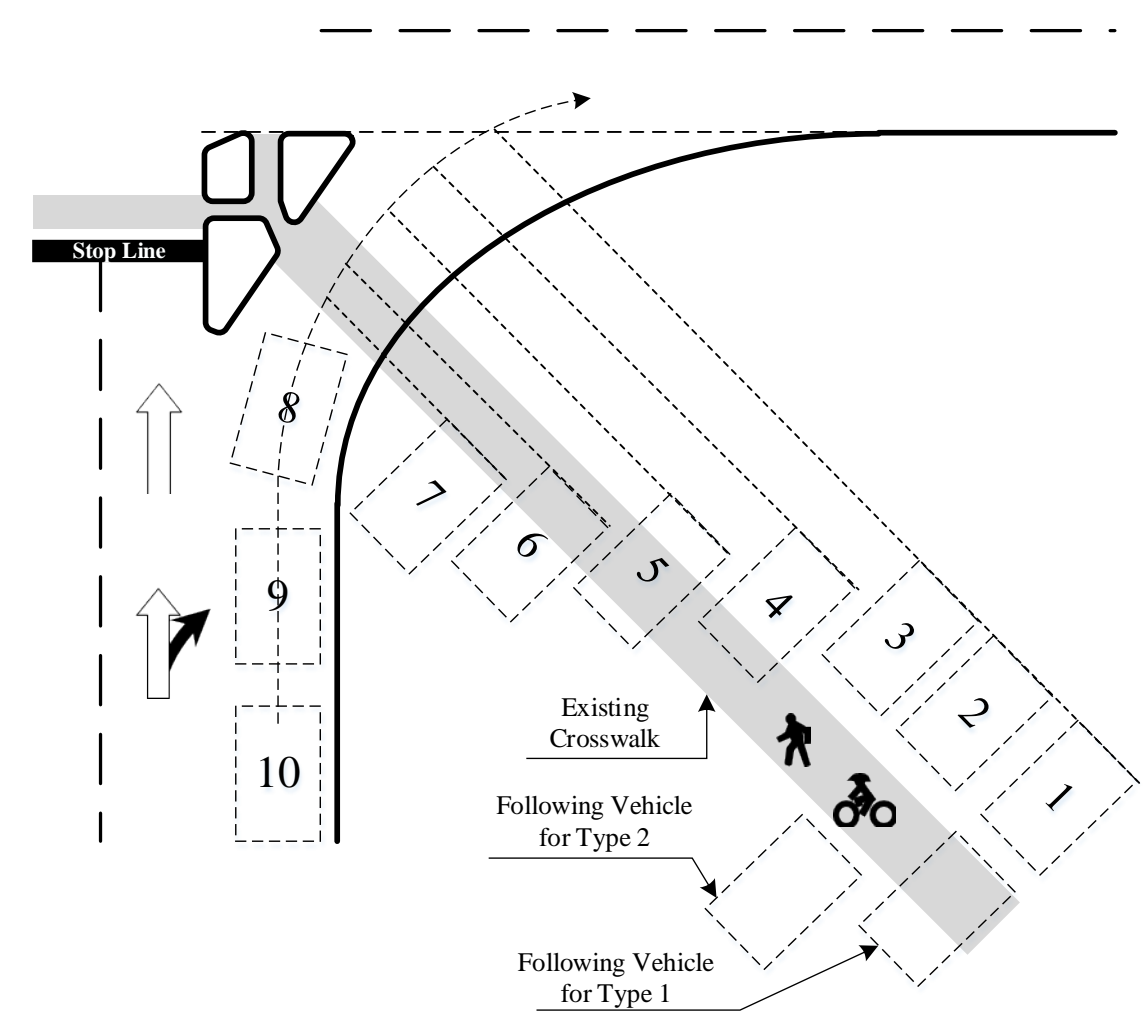

Figure 3. Ten types of observed stopping positions of right-turn vehicles in relation to the crosswalk.

Table 1. Multiple stops of the four observed sites.

\begin{tabular}{ccccc}
\hline Site & EB & SB & NB & WB \\
\hline Number of vehicles with multi-stops & 70 & 15 & 104 & 67 \\
Total number of vehicles with stops & 652 & 660 & 239 & 244 \\
Vehicles performed multi-stops (\%) & $11 \%$ & $2 \%$ & $44 \%$ & $27 \%$ \\
\hline
\end{tabular}

Table 2. Pedestrian and bicycle behavior analysis.

\begin{tabular}{|c|c|c|c|c|c|c|c|c|}
\hline \multirow{2}{*}{$\begin{array}{l}\text { Behavior } \\
\text { Ped/Bike }\end{array}$} & \multicolumn{2}{|c|}{ EB } & \multicolumn{2}{|c|}{ SB } & \multicolumn{2}{|c|}{ NB } & \multicolumn{2}{|c|}{ WB } \\
\hline & $\mathrm{P}$ & B & $\mathrm{P}$ & B & $\mathrm{P}$ & B & $\mathrm{P}$ & B \\
\hline Waiting & 72 & 18 & 27 & 6 & 11 & 3 & 10 & 11 \\
\hline Stop and $\mathrm{go}^{(1)}$ & 42 & 4 & 30 & 3 & 9 & 1 & 3 & 3 \\
\hline Crossing at front & 16 & 4 & 21 & 18 & 40 & 7 & 8 & 13 \\
\hline Crossing at rear & 48 & 18 & 3 & 0 & 26 & 3 & 11 & 9 \\
\hline Give up & 0 & 0 & 0 & 0 & 1 & 0 & 0 & 0 \\
\hline Potential conflicts ${ }^{(2)}$ & 64 & 22 & 24 & 18 & 66 & 10 & 19 & 22 \\
\hline Potential conflicts \% & $16 \%$ & $31 \%$ & $16 \%$ & $15 \%$ & $38 \%$ & $59 \%$ & $29 \%$ & $35 \%$ \\
\hline Total ped/bike potential conflicts \% & \multicolumn{2}{|c|}{$18 \%$} & \multicolumn{2}{|c|}{$16 \%$} & \multicolumn{2}{|c|}{$40 \%$} & \multicolumn{2}{|c|}{$32 \%$} \\
\hline Total volume & 398 & 70 & 147 & 120 & 174 & 17 & 65 & 63 \\
\hline
\end{tabular}

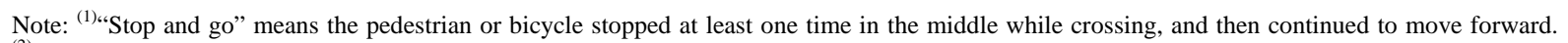
(2)“Potential Conflicts” is a sum-up of all ped/bike crossings at front or rear of the vehicle due to the lane-block, the situation of "give-up” is also counted as potential conflicts. 


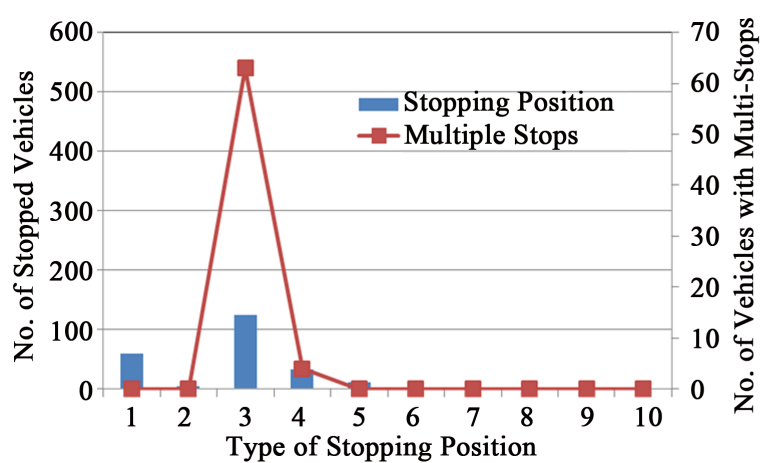

(a)

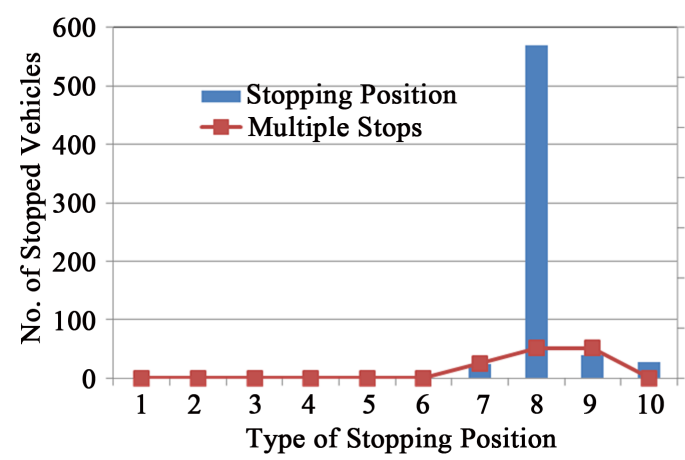

(c)

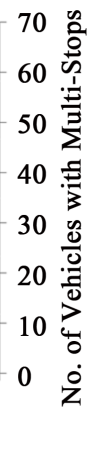

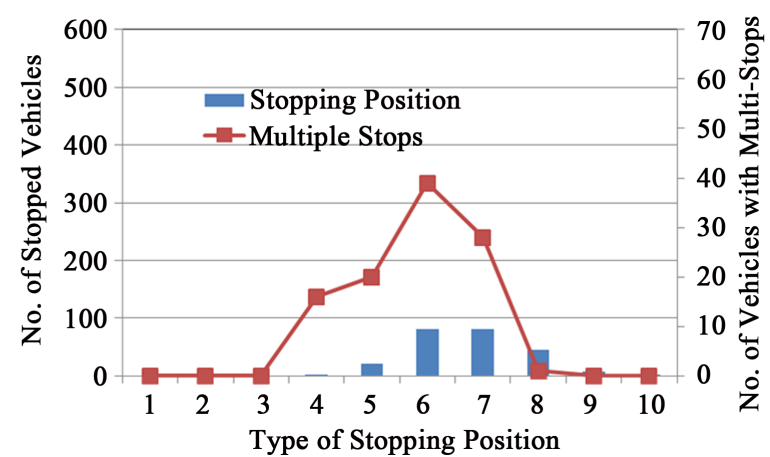

(b)

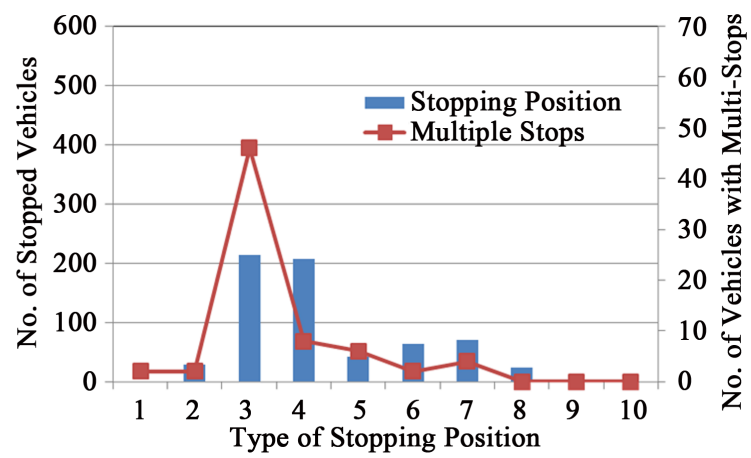

(d)

Figure 4. Types of stopping positions distribution of the four observed sites. (a) WB; (b) NB; (c) SB; (d) EB.

is not reflected in Table 2. In particular for NB, the bicyclists might encounter conflicts with the right-turn vehicles by $59 \%$ (10 out of 17 bicycles). Similarly for WB, there were $29 \%$ and $35 \%$ for pedestrians and bicyclists, respectively to encounter conflicts with right-turn vehicles, while their demands were relatively smaller than the others, 65 and 63, respectively. Similar results were found for the EB for bicycles as well. Besides, for the EB, while the pedestrians' demand increased to 398, the potential conflicts decreased to $16 \%$. It seems that the crossing demand is inversely proportional to the potential conflicts at the right-turn channels for the EB, NB, and WB.

However for SB where there was no crosswalk marked, the lowest potential conflicts (16\% for pedestrians and $15 \%$ for bikes) were observed based on a medium size of crossing demand (147 pedestrians and 120 bikes). Whether there is a specific space for crossing, may affect the potential conflicts as well.

Further, Table 3 tells that the higher demand of right-turn vehicles doesn't result in the higher potential conflicts. For instance for SB where the total stopped right-turn traffic volume was the highest (660 vehicles), only $4 \%$ of the vehicles blocked the crosswalk. On the contrary, $78 \%$ of the right-turn vehicles for the NB occupied on the crosswalk within the right-turn channel. It is more likely that the relationship between the demand of right-turn vehicles and the potential conflict was quite weak.

Therefore it is the potential conflicts, rather than the demand that more affect the stopping positions of right-turn vehicles. The stopping positions are mostly determined by whether there is a: 1) sufficient sight of view; 2) guidance for Optimized Stopping Position (OSP), which is defined as the position where a vehicle can most efficiently complete its turning movement.

If there is a kind of indication such as a painted box displaying on the pavement along the right-turn channel for proper stopping positions of right-turn vehicles, the conflicts could be greatly reduced. This motivates the idea of RIGHT-TURN VEHICLE BOX (RTVB) as a supplementary right-turn safety treatment, which should (1) provide sufficient sight distance; (2) offer clear guidance for optimized stopping position; and (3) clear the crosswalk to ensure smooth crossings of ped/bike. This is similar to the already developed Bike Box (BB) [19] [20], typical applications of which include the red BB in Main Street and Union Street in Vancouver, British Columbia, Canada, and the green BB in England [21]-[23]. 
Table 3. Comparison of right-turn stopping vehicles and ped/bike potential conflicts.

\begin{tabular}{rcccc}
\hline & EB & SB & NB & WB \\
\hline Total stopping right-turn vehicles blocking crosswalk & 384 & 24 & 186 & 49 \\
Total stopped right-turn volume & 652 & 660 & 239 & 244 \\
Percent vehicles blocking crosswalk & $59 \%$ & $4 \%$ & $78 \%$ & $20 \%$ \\
\hline
\end{tabular}

Remark: Type 4 through 7 is counted as types that would block the crosswalk (Figure 3).

\section{Methodology}

\subsection{Problem Illustration}

Figure 5(a) is an illustration of the problem that might be encountered by a right-turn vehicle at a channelized intersection.

In Figure 5(a), $V_{C}$ represents the crossing vehicle, which is the first vehicle at the stop line on EB. The critical visibility distance for vehicle $\mathrm{V}_{\mathrm{C}}$ may be much farther back than point $\mathrm{A}$, and there is no any obstacle to block in the middle. $V_{T}$ is the through vehicle at the stop line along the approaching lane on NB. $V_{R}$ is the right-turn vehicle driving from the NB entering the right-turn channel on Red.

In this situation, $V_{C}$ and $V_{T}$ are static, and already stop at the stop lines. $A$ is the upper right point of $V_{C}$, $R_{1}$ represents the driver's eye in $V_{R}$ (also as a reference of the stopping position for $V_{R}$ ), and $B$ is the upper right point of $\mathrm{V}_{\mathrm{T}}$.

Assuming that the drivers stop at $\mathrm{R}_{1}$ after they enter the channel as indicated in Figure 5(a), there are two possible scenarios under such situation: (1) right-turn with blocked view; and (2) right-turn away from OSP.

\section{Scenario 1: Right-turn with blocked view}

In Figure 5(a), the line $\mathrm{AR}_{1}$ connects the vehicle on crossing street and the driver's eye within the right-turn vehicle. There is an object $\mathrm{V}_{\mathrm{T}}$ (could be a vehicle or other objects, such as tree or telegraph poles in other cases) blocking the driver's view at $R_{1}$. Under such situation, the driver cannot see completely the traffic from the crossing street (EB in Figure 5(a)), at least cannot see $V_{C}$ which is on the far right end and directly blocked by $V_{T}$. In order to get a clear view for decision making, the driver needs to drive from $R_{1}$ to $R_{2}$ (the second stop happens here) to watch the vehicle on the far right lane.

As illustrated in Figure 5(a), the line $A B$ can be viewed as the lower boundary of the visible area for $V_{R}$ when the obstacle $V_{C}$ exists. In other words, all the area on the upper side of line $A B$ is visible to $V_{R}$. Therefore, if a vehicle stops on the upper area of the boundary, the driver can obtain the required view at one time without extra movement to make the decision.

If a right-turn vehicle stops below the boundary, part of the traffic condition would not be available for the driver. In order to make a correct turning decision, the driver needs to execute the second or even third stop for sufficient view.

The above discussion is based on the assumption that $\mathrm{V}_{\mathrm{C}}$ is in the static status, and it locates at the most difficult position for $V_{R}$ to observe. If $V_{C}$ is moving when $V_{R}$ enters the channel (for example, already in A' as indicated), then with the movement of $V_{C}$ to the right (east), line $A B$ begins to move leftwards, which broadens the view of $V_{R}$. In other words, $V_{R}$ has to move to $R_{2}$ before it can successfully see $V_{C}$, but at the location $A$ ', even when $V_{R}$ locates at the position $R_{1}, V_{R}$ can see $V_{C}$ at point $A^{\prime}$ '.

Based on the above discussions, it is suggested that the vehicle box be placed at the visible area in Figure 5(a) above the lower boundary to provide one time clear vision to drivers. In addition, the location of the vehicle box should also consider: (1) the safe distance (H) from vehicle's front bump to the edge of the road in Figure 5(a), which can also be viewed as the upper boundary of the RTVB; and (2) the clearance of way for ped/bike crossing if any.

\section{Scenario 2: Right-turn away from OSP}

If there is no blocking object along the line $\mathrm{AR}_{1}$ in Figure 5(a), the driver at $\mathrm{R}_{1}$ should have a clearer view on all vehicles coming from the eastbound (EB), and make their decisions accordingly. In this case, there should theoretically be only one stop for right-turn vehicles. However, some drivers tend to double examine the traffic for a proper gap to enter on real-time basis when driving along the channel. In other words, these vehicles would slowly yield and even have multiple short stops before they reach their comfortable points to merge into the 


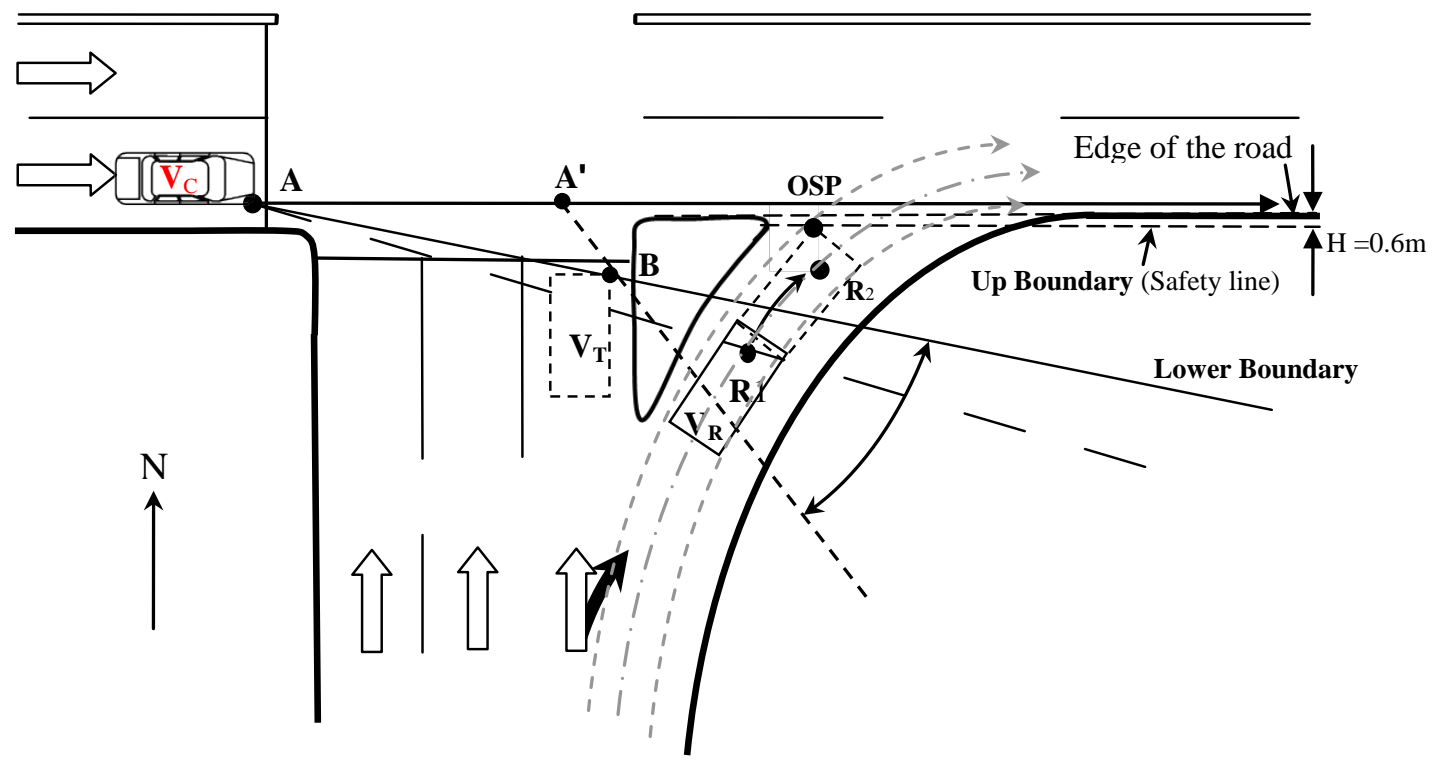

(a)

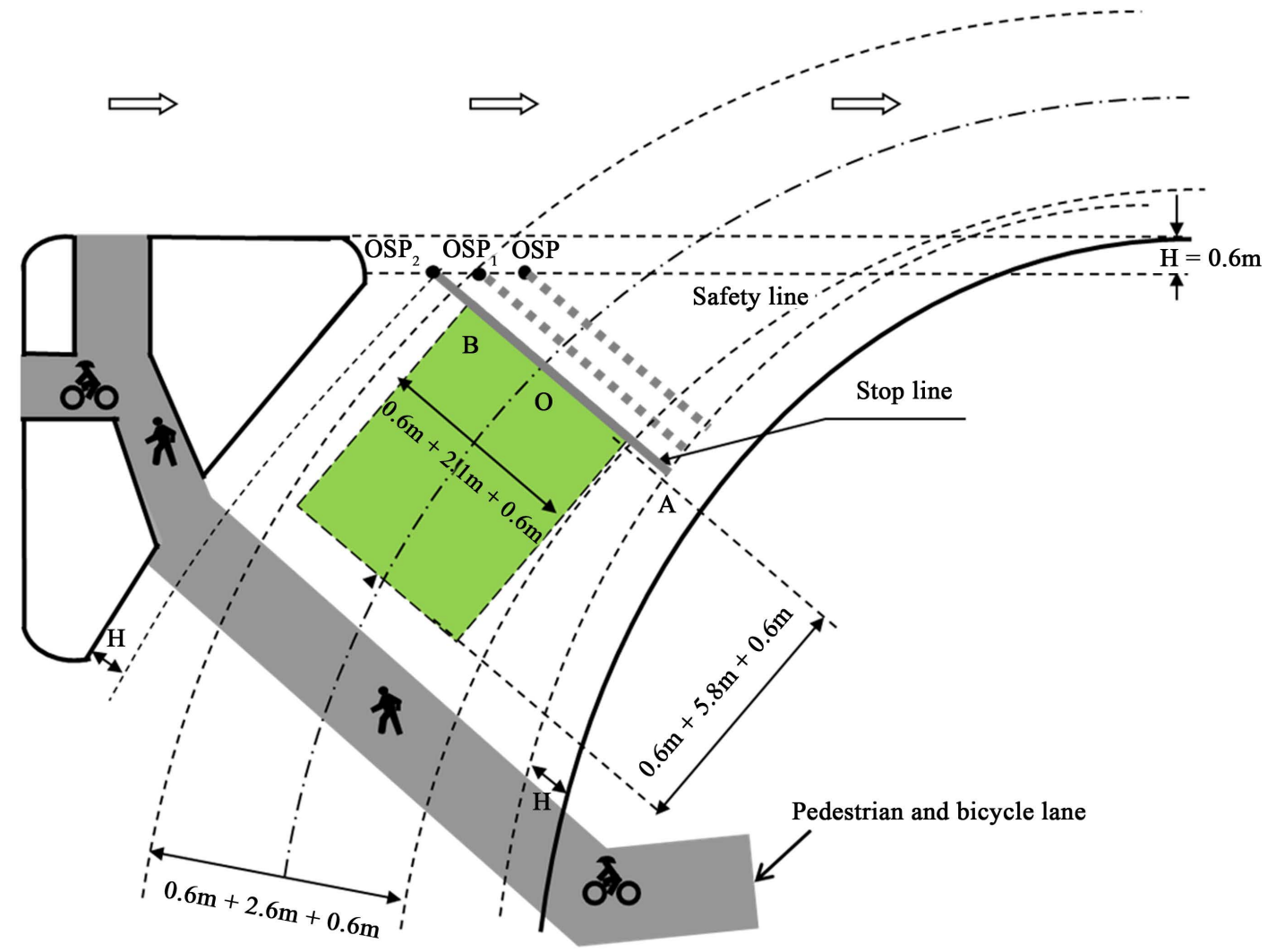

Note: This figure is not to scale

(b)

Figure 5. The design of a RTVB. (a) A right-turn vehicle at the channel; (b) The right-turn vehicle box and the crosswalk. Note: (1) OSP: original point for passenger car but not suitable for setting stop line for other vehicle. (2) OSP $\mathrm{O}_{1}$ : this point is for setting stop line for big vehicle (bus, trucks). But this point is not good enough because when the huge vehicle stop, the front of the vehicle will still cross the $\mathrm{H}$. (3) $\mathrm{OPS}_{2}$ : this point is the final point for determine the box and stop line. It considers the safety for both passenger car and huge vehicles. 
crossing street. Even though multiple stops may be required to both comply with Texas law and likely correspond with fewer conflicts with crosswalk users, one of the engineers' design objectives should be to try their best to eliminate during the design stage, the unnecessary number of stops and/or yields for all vehicles including right turn vehicles.

Under such a situation, a guide to the most OSP is necessary. Assuming that the minimum safe distance is $\mathrm{H}$ in Figure 5(a), vehicles should stop behind the safety line for sure of being safe. Therefore, when the upper left corner of the vehicle reaches the safety line, the vehicle has reached its last feasible position to stop before merging. This position provides right-turn vehicles with the shortest distance and the most efficient route to enter the crossing road, and thus is the OSP for right-turn vehicles. The OSP in each right-turn channel can be determined by the following steps.

Step 1: Find out the edge of the crossing road the right-turn vehicle tends to enter; draw a safety boundary line using a recommended safe distance $\mathrm{H}$ (in AASHTO Green Book (15), $\mathrm{H}=0.6 \mathrm{~m}$ );

Step 2: Draw the trajectory of the right-turn vehicle (the axle of the vehicle) along the right-turn channel curve;

Step 3: Assume a vehicle size: $\mathrm{W}$ as the width and $\mathrm{L}$ as the length. When the upper left point of the vehicle hits the safety line, the intersecting point is determined as the OSP.

After the OSP has been determined, it is suggested that a vehicle box be positioned according to OSP (with OSP being the upper left point of the box). In addition, the location of the vehicle box should also ensure the clearance for crosswalk if any.

\subsection{Design of RTVB}

\subsubsection{RTVB Position Design}

A vehicle box is supposed to accommodate at least one typical passenger car. According to the AASHTO Green Book (15), the width of one typical passenger car in design is $2.1 \mathrm{~m}$ and the length is $5.8 \mathrm{~m}$. However, the box size cannot be simply designed exactly as the size of this typical passenger car, because, although at most sites the majority type of vehicles are passenger cars, the design should also consider the needs of heavy duty vehicles (HDV).

In order to meet the design need for HDV, which typically have a size of $2.6 \mathrm{~m}$ in width (15), the calibrated

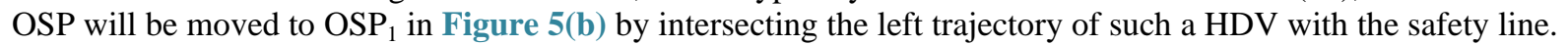
However, considering that the head of the heavy vehicle may stretch out and overpass the safety line, it is suggested that $\mathrm{OSP}_{1}$ be further calibrated.

To make sure that a HDV will not overpass the safety line when they follow the guidance of RTVB, the leftmost boundary line can be defined by leaving a safety margin $\mathrm{H}(0.6 \mathrm{~m}$ by the Green Book (15)) from the curve of the island(s). This leftmost boundary will intersect with the safety line at $\mathrm{OSP}_{2}$, and this point will be used as the final to determine the position of RTVB.

In Figure 5(b), the line $\mathrm{OSP}_{2}$-A is parallel to the axles of the turning vehicle. The line $\mathrm{OSP}_{2}$-A intersects the rightmost boundary (the parallel curve $0.6 \mathrm{~m}$ away from the channel curve) at $\mathrm{A}$. $\mathrm{O}$ is the center point of $\mathrm{OSP}_{2}-\mathrm{A}$, and $\mathrm{B}$ is the left up corner of the RTVB. $\mathrm{OB}=0.6 \mathrm{~m}+2.1 \mathrm{~m} / 2$. The position of RTVB can be determined by OB.

\subsubsection{RTVB Size Design}

For the size of RTVB, it is suggested to be $3.3 \mathrm{~m}(=0.6 \mathrm{~m}+2.1 \mathrm{~m}+0.6 \mathrm{~m})$ by $7.0 \mathrm{~m}(=0.6 \mathrm{~m}+5.8 \mathrm{~m}+0.6 \mathrm{~m})$ as is shown in Figure 5(a). This size is designed to accommodate a passenger car (2.1 m by $5.8 \mathrm{~m}$ ) plus a $0.6 \mathrm{~m}$ buffer on each side.

\subsubsection{Other Considerations}

(a) Dashed line or similar is suggested for left, right and rear boundary of the RTVB.

The box size is designed according to the size of a passenger car, which takes the largest population of the turning vehicles in most of cases. By using dashed lines or similar (as long as such design will not conflict with MUTCD standards and guidelines), the less HDV could also use the RTVB as a reference when turning.

(b) Colored Box can be used to attract the attention of both vehicles and ped/bike.

In some previous implementation of the Bike Box, filling the box with color has very positive effect (22). This idea could also be considered for the RTVB during implementation. Even though the all-filled green color 
box is used as an example in Figure 5(b), detailed designs still need to be further tested through simulation and field validation. Advanced studies on this are recommended so that both motor vehicle drivers and crosswalk users can correctly identify and react to the identified color and shape. Existing practices and standard on crosswalk treatments for roundabouts could be one of the references (e.g. [23]).

(c) The crosswalk should be well designed to accommodate the RTVB

The crosswalk should avoid the identified box area. At most of the cases such as illustrated in Figure 5(b), the crosswalk could be placed at the upstream of the right-turn vehicle trajectory with no direct intersection with the box. Other possibility could be to place the crosswalk outside the queuing location for stopped turning vehicles, depending on the geometrical possibilities.

In Figure 5(b), the gap between the RTVB and the crosswalk should ensure a minimum safety margin, which should be at least at a distance of $\mathrm{H}(0.6 \mathrm{~m}$ as indicated in the Green Book [15]). On the other hand, the crosswalk should not be too far from the RTVB to avoid being interrupted by its following vehicle within the rightturn lane.

\subsubsection{Suggested Implementation Procedure for RTVB}

The implementation of the RTVB could follow a nine-step procedure.

Step 1: Set up the upper boundary for the RTVB

The upper boundary is the safety line $0.6 \mathrm{~m}$ from the edge of the road (indicated in).

Step 2: Set up the lower boundary for the RTVB (Figure 5(a))

Line $A B$ is regarded as the lower boundary for vision check (Figure 5(a)). The line can be defined by connecting the upper right point of $\mathrm{V}_{\mathrm{C}}$ and the upper right point of $\mathrm{V}_{\mathrm{T}}$. The area above this lower boundary is considered as visible to the drivers.

Step 3: Area check

The area between upper and lower boundary within the channel is the potential area for RTVB. Whether a RTVB can be applied first depends on if there is a sufficient area to hold it.

Step 4: Plot trajectory for leftmost and rightmost boundary

The leftmost and rightmost boundary can be plotted by drawing parallel curves $0.6 \mathrm{~m}$ away from the curb island and the channel, respectively.

Step 5: Locate $\mathrm{OSP}_{2}$

$\mathrm{OSP}_{2}$ can be determined by intersecting the leftmost boundary line with the safety line (Figure 5(b)).

Step 6: Determine the stop line

Stop line (Line $\mathrm{OSP}_{2}$-A in Figure 5(b)) is the tangential line to the axle of the vehicle trajectory in the channel. The line starts at $\mathrm{OSP}_{2}$.

Step 7: Draw the RTVB

Finding out the O and calculate OB (Figure 5(b)). Draw a box of $3.3 \mathrm{~m}$ by $7.0 \mathrm{~m}$ or a size that can accommodate the largest population of the right vehicles with safe buffer allowed on each side. The left, right, and rear boundary of the box is suggested being in an open form by plotting the dashed lines for the less vehicles with larger sizes.

Step 8: Design crosswalk

The crosswalk is suggested to be at least $0.6 \mathrm{~m}$ away from RTVP to ensure the safety, and should not be too far from the box to avoid the interruption by the second vehicle in the channel.

Step 9: Other construction and management issues

Other issues could include: (a) ped/bike signal timing and devices adjustments if needed; (b) refuge islands reconstruction if needed; and (c) other considerations that might be necessary.

\section{Crosscheck with Existing Field Observations}

The proposed idea on the right-turn vehicle box was crosschecked with the stopping positions within the rightturn channel in the four observation sites mentioned earlier. The traffic volume and vehicles' right-turn movement recorded during four typical weekdays (Monday to Thursday), from $7 \mathrm{am}$ to $7 \mathrm{pm}$, were selected.

\subsection{Daily Traffic and Ped/Bike Volume on the Spot}

The daily traffic and ped/bike volumes on the spot are tabulated in Table 4. 
Table 4. Daily traffic volume (7 am to $7 \mathrm{pm}$ ) along all approaches.

\begin{tabular}{ccccccccc}
\hline & \multicolumn{2}{c}{ EB } & \multicolumn{2}{c}{ SB } & \multicolumn{2}{c}{ NB } & \multicolumn{2}{c}{ WB } \\
\cline { 2 - 9 } & Volume & $\%$ & Volume & $\%$ & Volume & $\%$ & Volume & $\%$ \\
\hline Left-turn vehicle & 7654 & $12 \%$ & 8651 & $14 \%$ & 10,467 & $14 \%$ & 15,542 & $22 \%$ \\
Through vehicle & 47,202 & $74 \%$ & 46,565 & $76 \%$ & 60,556 & $81 \%$ & 48,038 & $69 \%$ \\
Right-turn vehicle & 8930 & $\mathbf{1 4 \%}$ & 6379 & $\mathbf{1 0} \%$ & 3738 & $\mathbf{5 \%}$ & 6358 & $\mathbf{9 \%}$ \\
Total motor vehicle volume & $\mathbf{6 3 , 7 8 6}$ & $100 \%$ & $\mathbf{6 1 , 5 9 5}$ & $100 \%$ & $\mathbf{7 4 , 7 6 1}$ & $100 \%$ & $\mathbf{6 9 , 9 3 8}$ & $100 \%$ \\
Pedestrian & 398 & N/A & 147 & N/A & 174 & N/A & 65 & N/A \\
Bicycle & 70 & N/A & 120 & N/A & 17 & N/A & 63 & N/A \\
\hline
\end{tabular}

In Table 4, there were 14\%, 10\%, 5\% and 9\% right turn vehicles at the EB, SB, NB and WB directions, respectively. Also observed is that there is a certain portion of ped/bike volume at this intersection pair, which is not often observed in most of the intersections in Houston area.

\subsection{Motor Vehicle Multi-Stop Analysis}

By following the nine-step implementation procedure of the RTVB, the RTVBs were proposed on the four layouts of selected sites in Figure 6. The designs of RTVBs were compared with the current site designs.

According to the data observed, the SB has the fewest multi-stops, while the NB has the biggest multi-stop rate. Given that the concept of RTVB is correct, it can be inferred that the more the first stopping position of the most vehicles is close to RTVB, the fewer stops they will have before they finish their turn. In other words, if a vehicle's first stop is within the RTVB, it is expected that this vehicle can efficiently finish its turns without extra stops.

As mentioned before, the most frequently stopped position (MFSP) located in type 3 for the EB and WB, in type 6 and 7 for the NB, and in type 8 for the SB. When comparing the position of the RTVB and the MFSP for each site, it is found that the SB has the best match with these two positions. Stopping location of Type 8 is very close to the designed RTVB in Figure 6, which means that, most drivers have their first stops at a safe and efficient place with no hesitation when making turnings. The NB is another example, but on the opposite site. According to the observation, the MFSP of this site differs most with the RTVB while compared with the other three sites. The figure of its multi-stop rate turns out to be the highest among the four sites, which also confirms with the theory of RTVB.

\section{Simulator Test}

\subsection{Test Scenarios}

A driving simulator test was carried out to examine the impacts of the RTVB on drivers' stopping positions for right turn. Three different scenarios were designed for comparison purpose, which is illustrated in the virtual track in Figure 7. During the test, subjects encountered a red light and a conflict with pedestrian and a vehicle coming from left side at each of the three signalized intersections. Except for these locations, they were able to travel in a free flow mode. They were supposed to make right-turns within the designed channels of the three intersections, where subjects experienced no RTVB (Scenario 1), a blank RTVB (Scenario 2) (i.e. RTVB with white frame only but no color filling), and a RTVB filled with green color, respectively. Specifically, subjects started from "Start Point 1" to "Start Point 2" for scenario 1, from "Start Point 2" to "Start Point 3" for scenario 2, and from "Start Point 3" to "Return to Point 1" for Scenario 3.

Vehicle’s activities were only measured during right-turn movement at the three intersections marked with scenario numbers. The forth intersection marked with no RTVB serves as a connector for a continuous test of all three scenarios and was not counted for measurement. The distance between adjacent intersections was equal to each other, approximately $200 \mathrm{~m}$. 


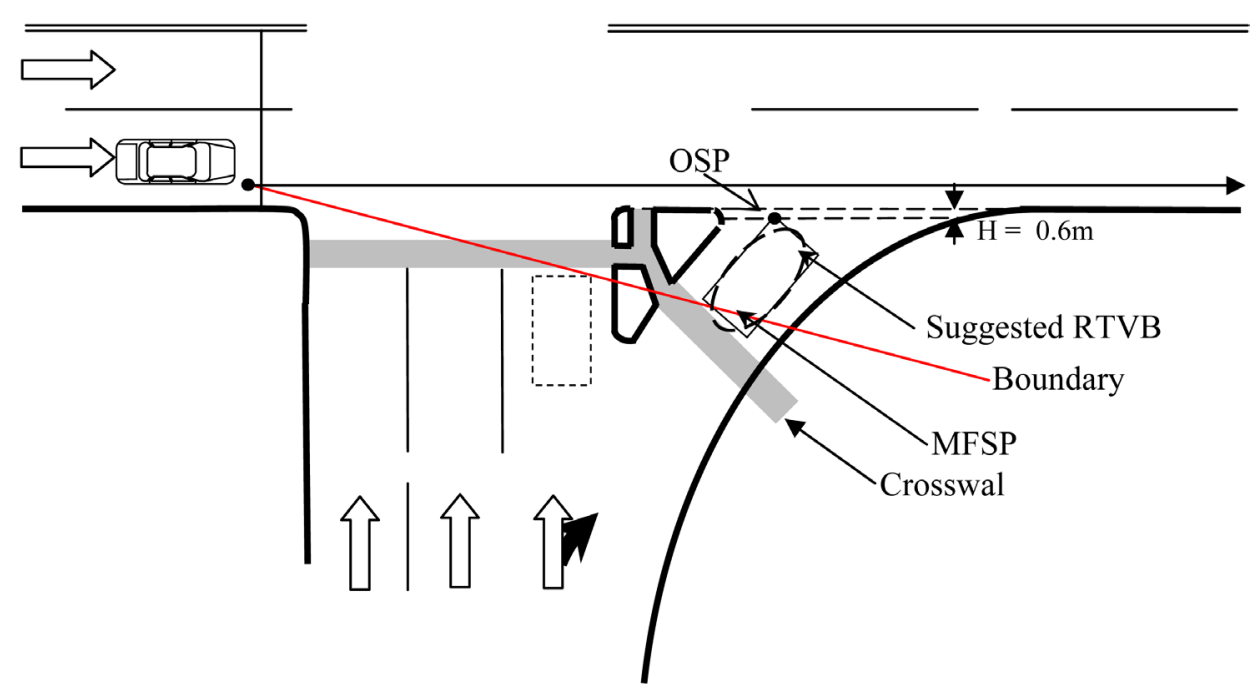

(a)

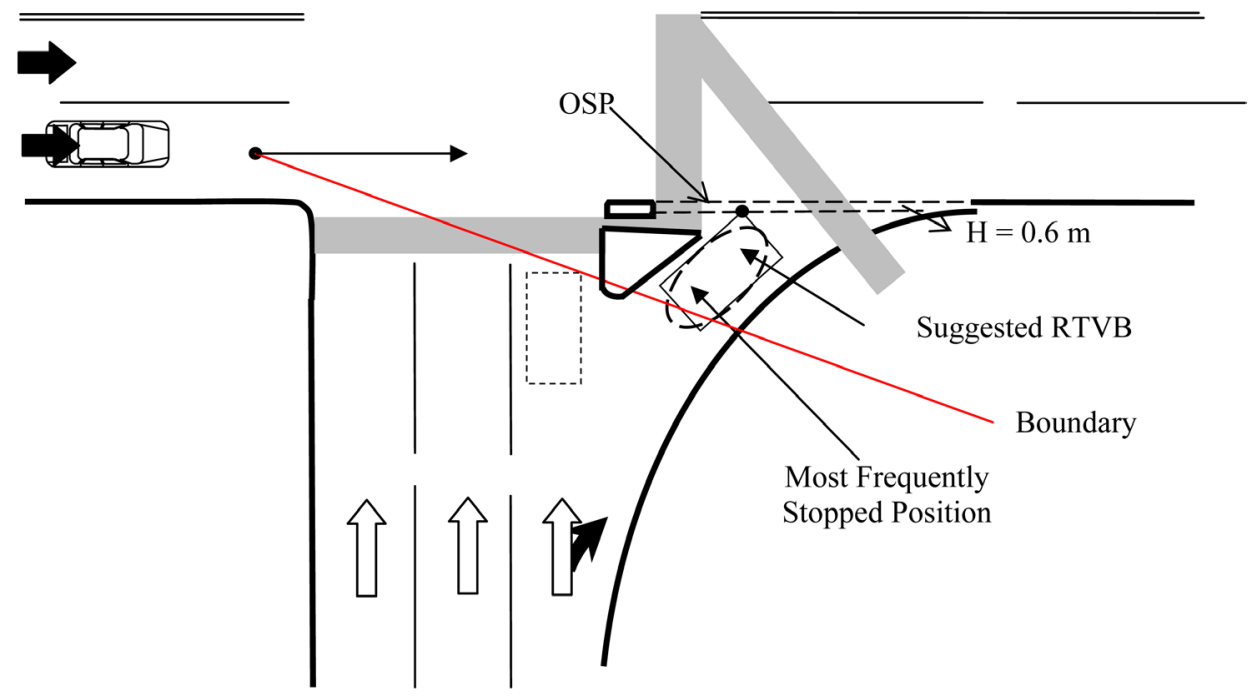

(b)

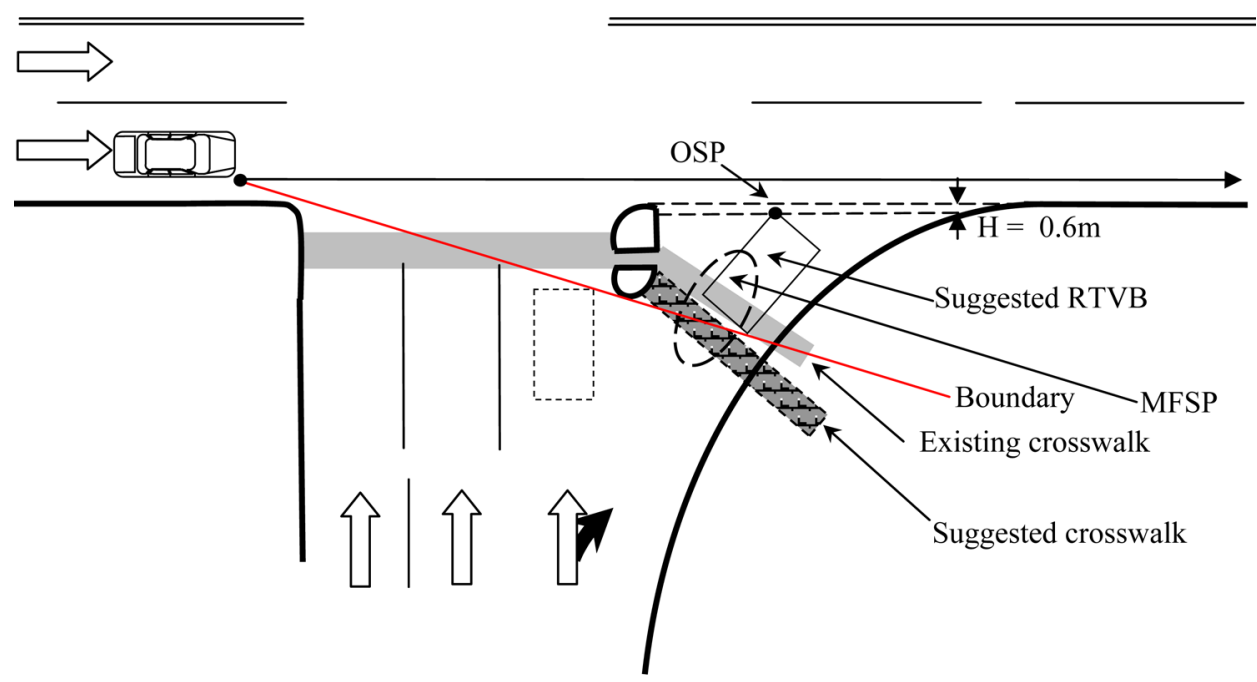

(c) 


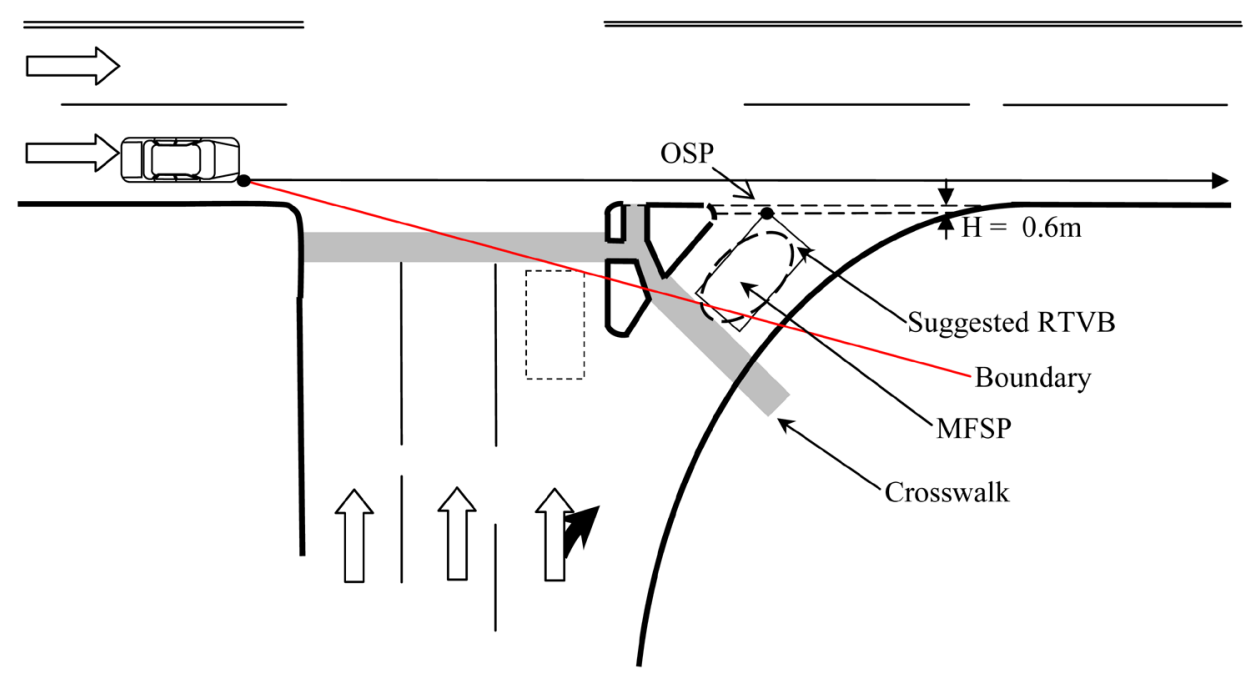

(d)

Figure 6. Most frequently stopped positions and suggested RTVB for four bounds of the coupled intersection. (a) The WB; (b) The SB; (c) The NB; (d) The EB.

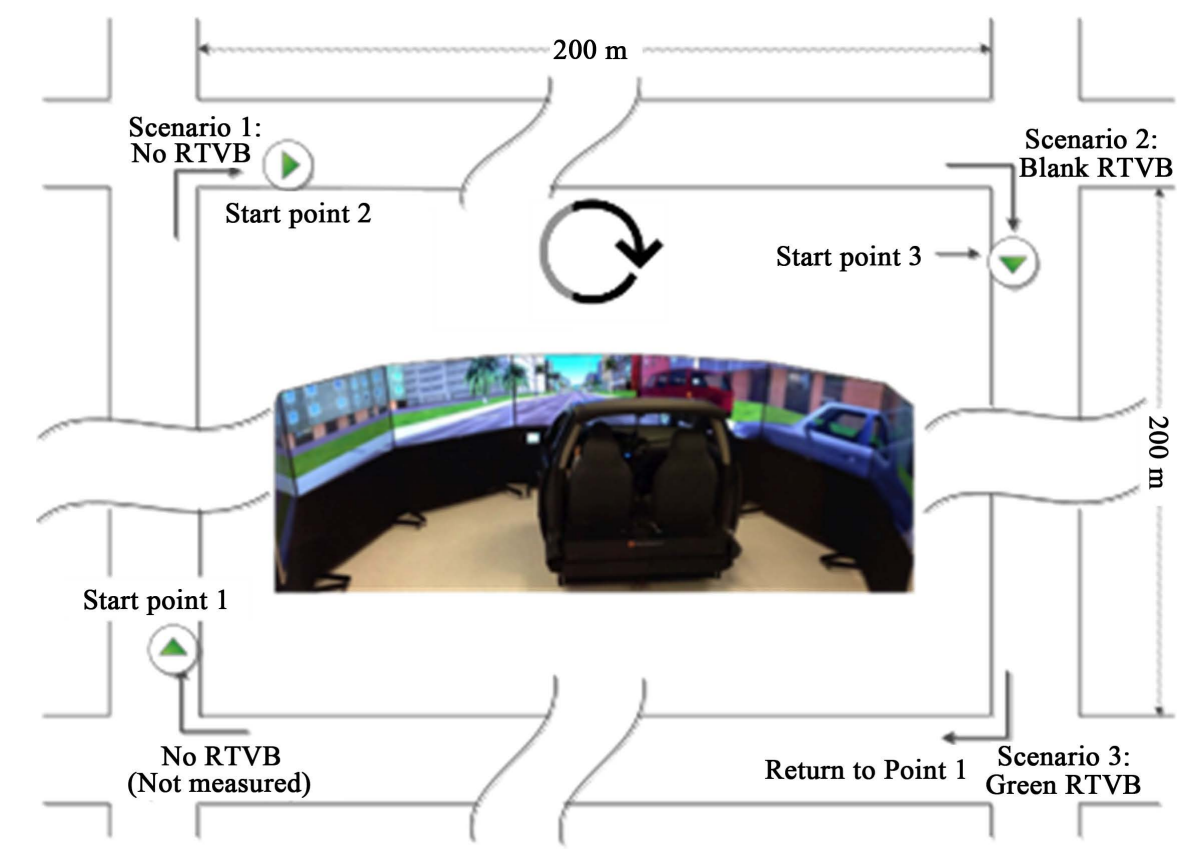

Figure7. Driving simulator test track with three starting points and three scenarios.

\subsection{Test Procedure}

Twenty-one subjects were recruited (18 males and 3 females), all possessing valid $C$ driver licenses and aging between 18 and 35 years old. Before formal tests, subjects obtained a short training course individually, including the introduction of RTVB, the right-turn on red policy, and the pre-test driving. During the training driving, subjects drove through a short segment to make two to three right turns, in order to be familiar with the simulation environment and the operation of the driving simulator.

In the formal tests, subjects randomly chose one of the three start points, and then drove through the entire virtual track in Figure 7. The same procedure went through for each subject, so as to eliminate the interference of scenario sequence. At each intersection, subjects needed to make a right turn during the red phase. They may encounter conflict with vehicles from the left on the crossing street. Raw data were collected at a frequency of 
$60 \mathrm{~Hz}$. The recorded information includes vehicle's geo-location ( $x, y$, and $z$ coordinates), speeds (m/s), and braking level. The braking level was measured by the intensity of pushing the brake pedal, ranging from 0.0 to 1.0. 1 value indicates fully stop, while 0 represents no intention of stop.

\subsection{Test Results}

The test results are plotted in Figure 8, where the configuration of each scenario is sketched on the left side, while the frequency diagram of stopping locations are placed on the right side. The stop locations in Figure 8 are measured as the front bump positions of vehicles.

For scenarios with the RTVB (Figure 8(b) and Figure 8(c)), subjects were supposed to stop within the box and not longer than 3 meters away from the stop line in front of RTVB. In this way, vehicles can stop as type 3 in Figure 3.

Figure 8(a) shows that, with no RTVB, about 20\% of vehicles' front parts encroached the crosswalk, which is spotted as type 4 to 7 in Figure 3. Sixty-five percent of vehicles stopped within 0 and 4 meters behind the stop line, and $13 \%$ of vehicles stopped far from the stop line, ranging from 8 to 12 meters.

Figure 8(b) and Figure 8(c) show that, when the RTVB is provided, all subjects stopped within the plotted box along the right turn channel. In Figure 8(b) for blank RTVB design, more than 65\% of the subjects stopped within the ideal area, whereas there is still about 35\% of them stopped at the type 4 to 7 (as are circled in Figure 8(b)).

When the green filled RTVB was provided in Figure 8(c), 100\% of the subjects stopped at the ideal area (i.e. type 3 in Figure 3). This implies that the RTVB with green color filling could be the best choice.

Significant difference in stopping positions was examined by the ANOVA single factor test, the results of which are shown in Table 5.

There was significant difference observed between Scenarios 1 and 2, which means that the blank box affected vehicle’s stopping positions significantly. A similar result was found when comparing Scenarios 2 and 3. This implies that vehicles' stopping positions were also subject to the color of box significantly.

\section{Conclusions}

This paper proposed a RTVB as a supplementary treatment to right-turn vehicle conflicts between right-turn vehicles and crossing vehicles as well as with ped/bike crossings.

In the field validation study, the southbound has the lowest multi-stop rate $2 \%$ (Figure 3 ). After examining the stopping locations, it is found that the most frequently stopped location for right-turn vehicles at this site is very close to the suggested RTVB. All other three sites have comparatively higher multi-stops rate in Figure 3 , which indicates an increase of potential conflicts and less efficiency right-turn operation. From this point of view, the RTVB could be a good way to improve the safety of right-turn movement.

In the meantime, the study of the ped/bike behavior shows that due to the lane-block or encroachment by some right-turn vehicles, ped/bike do encounter unsmooth crossing and face potential conflicts with right-turn vehicles. The placement of a RTVB could quickly guide the right-turn vehicles to the right place and clear the path for the crossings of ped/bike. The driving simulator test results indicate that the RTVB (especially the green filled RTVB) could properly guide vehicles to stop at the designed areas.

In all, the RTVB should be a promising treatment to enhance the safety and efficiency of right-turn operation. Meanwhile, it would also benefit the crossing of ped/bike.

Table 5. The ANOVA single factor results of significant difference in stopping positions.

\begin{tabular}{|c|c|c|c|c|c|c|c|c|}
\hline & Source of variation & SS & $\mathrm{df}$ & MS & $\mathrm{F}$ & $P$-value & F criteria & Significant \\
\hline \multirow{2}{*}{ Scenario 1 vs 2} & Between groups & $2.18 \mathrm{E}+02$ & 1 & $2.18 \mathrm{E}+02$ & $6.28 \mathrm{E}+00$ & $1.66 \mathrm{E}-02$ & $4.10 \mathrm{E}+00$ & \multirow{2}{*}{ Yes } \\
\hline & Within groups & $1.32 \mathrm{E}+03$ & 38 & $3.48 \mathrm{E}+01$ & & & & \\
\hline \multirow{2}{*}{ Scenario 2 vs 3} & Between groups & $2.72 \mathrm{E}+02$ & 1 & $2.72 \mathrm{E}+02$ & $1.06 \mathrm{E}+01$ & $2.41 \mathrm{E}-03$ & $4.10 \mathrm{E}+00$ & \multirow{2}{*}{ Yes } \\
\hline & Within groups & $9.77 \mathrm{E}+02$ & 38 & $2.57 \mathrm{E}+01$ & & & & \\
\hline
\end{tabular}



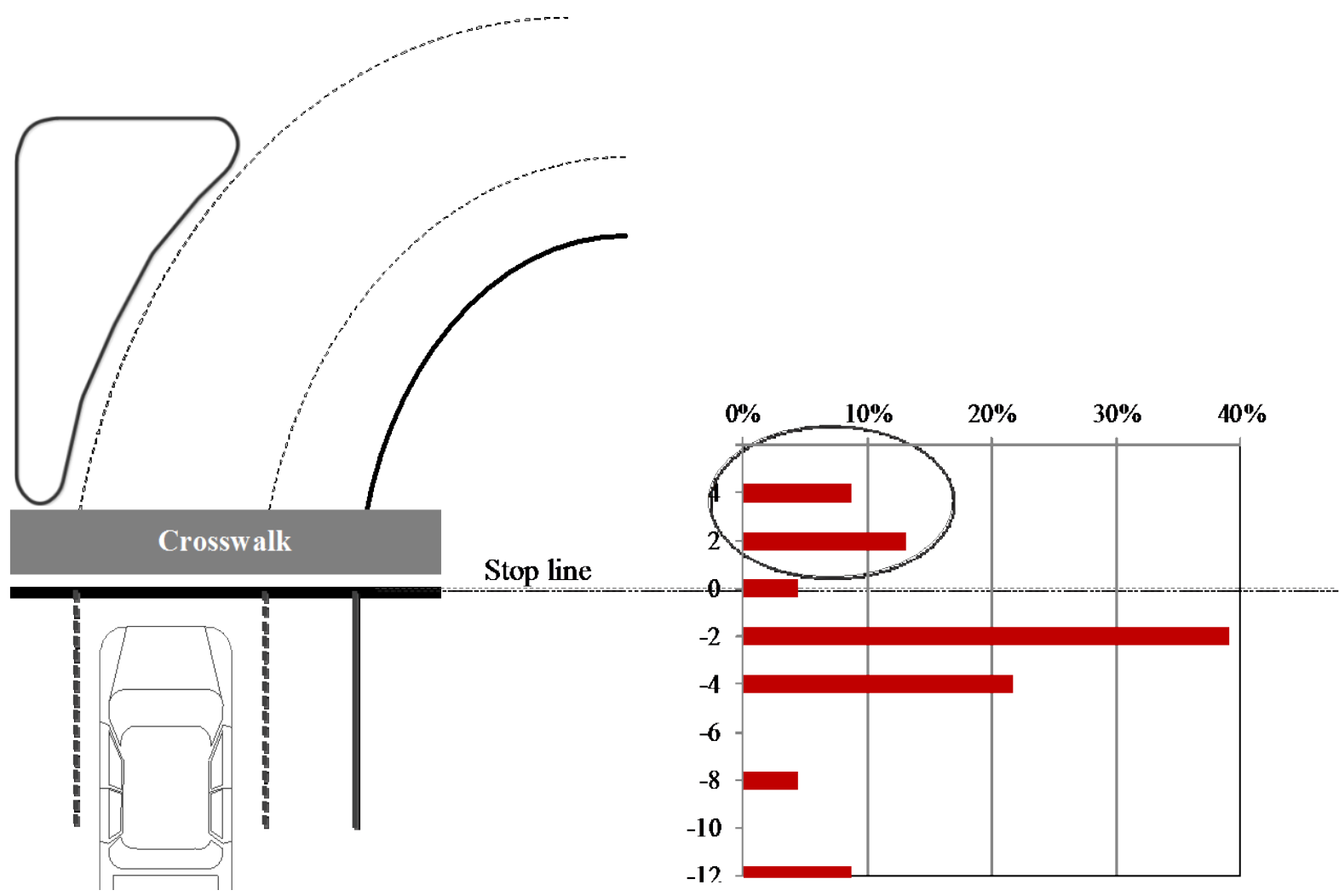

(a)

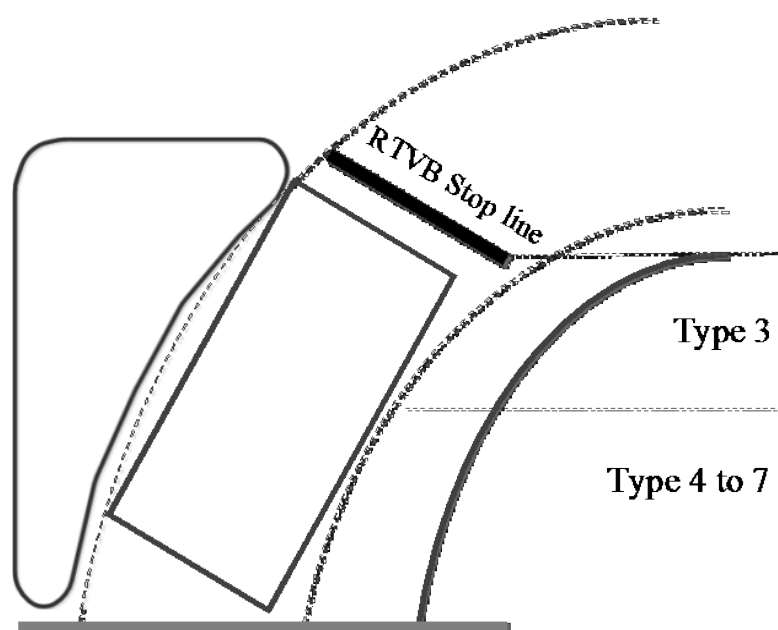

Crosswalk

Stop line
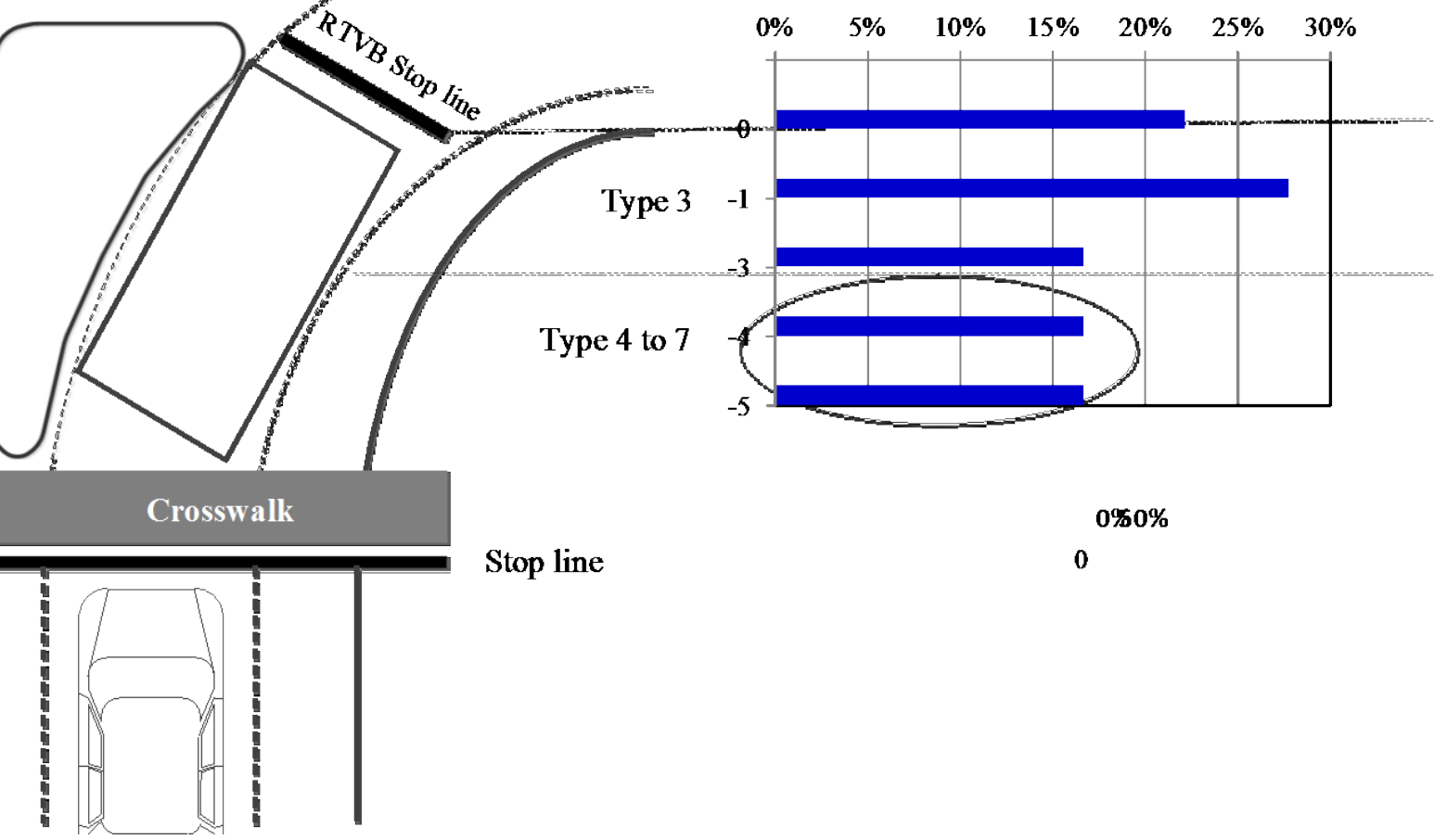

0\% 0\%

0

(b) 

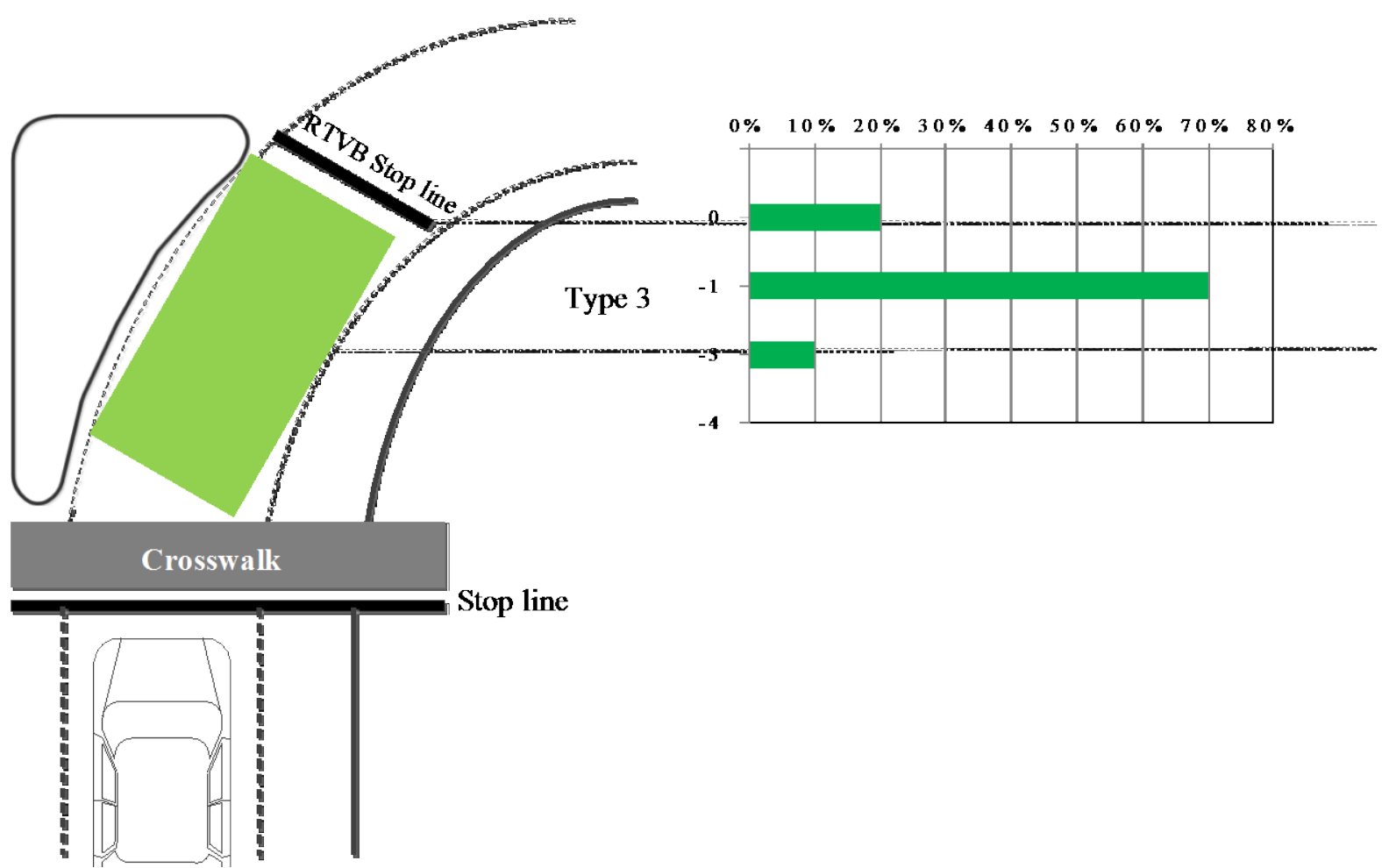

(c)

Figure 8. Simulator test results for three scenarios. (a) Test results for scenario 1 with no RTVB; (b) Test results for scenario 2 with blank RTVB; (c) Test results for scenario 3 with green RTVB.

Future work could focus on the completion of the warrants and design details, as well as the crossing benefits, i.e. to investigate whether a RTVB decreased conflicts with ped/bike. Implementation studies are encouraged to test the feasibility and further examine the idea. The issues to be studied include: (1) What could be the relative conflict or crash risk of the stopping locations in front of, on top of, or past the crosswalk due to operational, sightline, and human factors when designing a RTVB? (2) Should the change of turning vehicle in position and stop location impede or improve the movement of both turning vehicles and crosswalk users? (3) Moreover, could the concept of "Vehicle Box" be transplanted to many other places where turning needs assistance or improvements?

\section{Acknowledgements}

It is acknowledged that part of the ideas in this paper was inspired when conducting a research project sponsored by Texas Department of Transportation (TxDOT). This research is also supported in part by the Tier 1 University Transportation Center TranLIVE \# DTRT12GUTC17/ KLK900-SB-003, and the National Science Foundation (NSF) under grants \#1137732. The authors would like to express the gratitude to all those who directly or indirectly contributed to or supported this research. Special thanks go to Yijun Qiao for his hard work in preparing part of the computer program for data processing, and Ping Deng, Jun Wen, Albany Ng, and Rong Zhang for data processing. We also thank Elton Yang for participating in shaping the initial idea.

\section{References}

[1] National Highway Traffic Safety Administration (2013) 2012 Traffic Safety Facts: Research Note. Publication Number: DOT HS 811 856, November 2013. http://www-nrd.nhtsa.dot.gov/Pubs/811856.pdf

[2] National Highway Traffic Safety Administration (2013) 2012 Pedestrians Traffic Safety Fact Sheet. Publication Number: DOT HS 811888. http://www-nrd.nhtsa.dot.gov/Pubs/811888.pdf

[3] National Highway Traffic Safety Administration (2013) 2012 Bicyclists and other Cyclists Traffic Safety Fact Sheet. 
DOT HS 812018. http://www-nrd.nhtsa.dot.gov/Pubs/812018.pdf

[4] Zhou, H., Ivan, J.N. and Sadek, A.W. (2009) Safety Effects of Exclusive Left Turn Lanes at Unsignalized Intersections and Driveways. Transportation Research Board 2009 Annual Meeting, Washington DC, 30 January 2009. \#09-2000.

[5] Ozmen, O., Tian, Z. and Gibby, R. (2009) Safety of Special Time-of-Day Protected/Permitted Left-Turn Signal Control Display. Transportation Research Board 2009 Annual Meeting, Washington DC, 30 January 2009, \#09-2074.

[6] Lu, G.X. and Noyce, D. (2009) Intersection Signal Systems with Intelligent Pedestrian Accommodation: Dynamic Pedestrian Timing. Transportation Research Board 2009 Annual Meeting, Washington DC, 30 January 2009, \#09-3337.

[7] Liu, P., Lu, J., Chen, H. and Sokolow, G. (2008) Impacts of Separation Distances Between Driveway Exits and Downstream U-Turn Locations on Safety Performance of Right Turns Followed by U-Turns. Transportation Research Board 2008 Annual Meeting, Washington DC, 30 January 2008, \#08-0762.

[8] Texas Department of Transportation (2004) Regulations for Access Driveways to State Highways. Texas Department of Transportation, Austin, Texas, 2004.

[9] Qiao, F., Gampala, R. and Yu, L. (2010) Advanced Traffic Devices for Bicycles and Pedestrians to Friendly Cross Interchanges. Proceedings of the $17^{\text {th }}$ Intelligent Transportation System World Congress, Bushan, 25-29 October 2010, \#01324706.

[10] Alta Planning + Design (2005) Pedestrian and Bicycle Facilities in California, a Technical Reference and Technology Transfer Synthesis for Caltrans Planners and Engineers. July, 2005.

[11] Transportation Research Institute (1996) Right-Turn Lanes. Discussion Paper, Oregon State University, Salem, Oregon, No. 11.

[12] Zador, P.L. (1984) Right-Turn-on-Red Laws and Motor Vehicle Crashes: A Review of the Literature. Accident Analysis and Prevention, 16, 241-245. http://dx.doi.org/10.1016/0001-4575(84)90019-8

[13] Retting, P.L., Nitzburg, M.S., Farmer, C.M. and Knoblauch, R.L. (2002) Field Evaluation of Two Methods for Restricting Right Turn on Red to Promote Pedestrian Safety. ITE Journal, 72, 32-37. J

[14] McCoy, R.L., Bonneson, R.L., Ataullah, S. and Eitel, D.S. (1994) Guidelines for Right-Turn Lanes on Urban Roadways. Transportation Research Board TRB, National Research Council, Washington DC, Transportation Research Record 1445, 130-137.

[15] (2011) A Policy on Geometric Design of Streets and Highways. 6th Edition, American Association of State Highway and Transportation Officials (AASHTO), Washington DC.

[16] Pline, J.L., Ed. (1992) Traffic Engineering Handbook. Fourth Edition, Institute of Transportation Engineers, Prentice Hall, NJ.

[17] (1996) Intersection Sight Distance. National Cooperative Highway Research Program (NCHRP), Report 383.

[18] Texas Transportation Code, Chapter 545: Operation and Movement of Vehicles. http://www.statutes.legis.state.tx.us/docs/TN/htm/TN.545.htm

[19] (2008) Bikeway Planning and Design Guidelines Technical Appendix No. 1 Ottawa Cycling Plan. MMM Group \& Stantec in association with NOXON Associates, January 2008

[20] (2005) Bicycle Planning and Facility Design Best Practices. Sacramento Transportation \& Air Quality Collaborative, October 2005

[21] Jolicoeur, M. and Montreal, V.Q. (2005) Bicycle Access to Downtown Montreal: Investing in a Better Future. The Emerging Best Practices in Urban Transportation Planning Session of the 2005 Annual Conference of the Transportation Association of Canada, Calgary, 18-21 September 2005. http://conf.tac-atc.ca/english/resourcecentre/readingroom/conference/conf2005/docs/s9/Jolicoeur.pdf

[22] Office of Bicycle and Pedestrian Transportation (2004) Comprehensive Bicycle Plan. North Carolina Department of Transportation, Durham, NC.

[23] Baranowski, B. (2005) Pedestrian Crosswalk Signals at Roundabouts: Where Are They Applicable? Pedestrian Crosswalk Signals at Roundabouts, TRB Roundabout Conference, May 2005. http://www.teachamerica.com/roundabouts/RA057A_ppr_Baranowski.pdf 